\title{
Molecular Dynamics Study of Nanoimprint of Single Crystal Aluminium Thin Film
}

\author{
Y. Yuan, T. Sun, J.J. Zhang \\ Center for Precision Engineering, Harbin Institute of \\ Technology \\ Harbin, P. R. China
}

\author{
C. Liu \\ Center of Ultra-Precision Optoelectronic Engineering, \\ Harbin Institute of Technology \\ Harbin, P. R. China
}

\begin{abstract}
In current study, molecular dynamics simulations are performed to investigate nanoimprint processes on single crystal aluminium thin films with silicon stamp. An EAM potential developed for aluminium is adopted to describe the $\mathrm{Al}$-Al interaction in the aluminium thin film, and the Lennard-Jones potential is used to describe the $\mathrm{Al}-\mathrm{Si}$ atomic interaction. Simulations show that dislocation events are the main plastic deformation mode of $\mathrm{Al}$ thin film in nanoimprint. It is found that stamp size and stamp shape have significant influence on the dislocation events and imprint forces during the nanoimprint processes.
\end{abstract}

Keywords-nanoimprint; molecular dynamics; single crystal aluminium; thin film; stamp

\section{INTRODUCTION}

Nanoimprint lithography proposed by Chou [1] in 1995 has surmounted the resolution limitation of traditional photolithography, and has been proven as a revolutionary nanofabrication approach of low cost, high replication and high resolution. In the direct nanoimprint process for metal thin film, plastic deformation of materials determines the interior structural composition of the metal thin film, which consequently influences the macroscopical mechanical property, electrical property and optical property of the metal thin film. Therefore, it is needful to conduct fundamental research to investigate the deformation mechanism of metal during the nanoimprint process. However, the nanoimprint depth is typically only several or tens of atom layers in the metal thin film, which requires equipment with ultra-high resolution, and experimental investigation of the deformation mechanism is expensive and inconvenient currently. Furthermore, the nanoimprint process of metal thin film is a discrete process in essence, which is not appropriate to elucidate deformation mechanism with classical theory of macroscopic continuum mechanics. Molecular dynamics (MD) simulation has been proven as a powerful tool to elucidate deformation mechanism at nanometer scale in nanoimprint process due to its ability of providing atomic deformation details by tracking atomic motions.

Nowadays, many investigations have been conducted to study the nanoimprint process with the aid of MD simulations. Hsu et al. studied the effects of parameters, such as specimen temperature, punch velocity and spring back phenomena, on the nanoimprint process [2].They reported that the punch force decreases with increasing temperature, but increases with increasing punch velocity. And the spring back phenomena in horizontal direction are more serious than in vertical direction. Yao et al. investigated the effects of mold shape and sidewall roughness on the formation of atomic-scale structure in direct imprinting process on aluminium [3].The simulation results showed that with increasing taper angle, the required imprinting force is lower, and different formation mechanisms can be identified. The geometries of the imprint patterns were affected by sidewall roughness. Cheng et al. studied the effect of mold geometry, temperature, imprint velocity and water separation layer on nanoformed aluminium films [4-5]. They founded that a discontinuous geometry of the mold enlarged the effect of adhesion, the potential energy of $\mathrm{Al}$ film increased with imprint temperature, and its release was increasingly delayed with increasing imprint velocity. Water molecules buffered the mold and aluminium film, evaporation of water molecules caused pattern failure, and moderate water separation layers were beneficial for better ordered and more compacted pattern structures.

In this paper, we present MD simulations of the nanoimprint process of aluminium thin film with a silicon stamp, with which the deformation mechanism are elucidated. Furthermore, the effect of the stamp geometry on the nanoimprint process is studied.

\section{SIMULATION METHODS}

Fig.1 shows the MD model of nanoimprint of aluminium thin film with a silicon stamp. The embedded atom method (EAM) potential is adopted for simulating the interaction between aluminium atoms[6].For the $\mathrm{Al}-\mathrm{Si}$ atomic interactions between aluminium thin film and silicon stamp and $\mathrm{Si}-\mathrm{Si}$ atomic interactions in stamp, Lennard-Jones potential is used, and the detailed parameters can be found in our previous studies[7-8]. The aluminium thin film has a dimension of $12.15 \mathrm{~nm}, 8.1 \mathrm{~nm}$ and $12.15 \mathrm{~nm}$ in $\mathrm{X}, \mathrm{Y}$ and $\mathrm{Z}$ directions, respectively, and contains 73800 atoms. Periodic boundary condition is only applied along $\mathrm{X}$ and $\mathrm{Z}$ directions, and five atom layers at the bottom are fixed to restrict any rigid movement of aluminium thin film during nanoimprint process.

Before the simulation of nanoimprint process, the model is relaxed to its equilibrium through energy minimization. And then nanoimprint of the aluminium thin film is performed by 
using the silicon stamp. The silicon stamp is initially placed $1 \mathrm{~nm}$ above the surface of aluminium thin film, which exceed the truncation radius of Lennard-Jones potential to avoid the interaction between aluminium thin film and the silicon stamp before nanoimprinting. The nanoimprint process consists of loading and following retraction stages. In the loading stage, silicon stamp moves along negative $\mathrm{Y}$ direction with a constant speed of $100 \mathrm{~m} / \mathrm{s}$ to imprint into the aluminium thin film with a maximum imprint depth of $2 \mathrm{~nm}$. In the retraction stage, the silicon stamp move along positive $\mathrm{Y}$ direction with the same speed of $100 \mathrm{~m} / \mathrm{s}$.

Defect nucleation plays an important role in plastic deformation. In current study, common neighbour analysis (CNA) is utilised to distinguish the categories and positions of dislocations in the nanoimprint process.

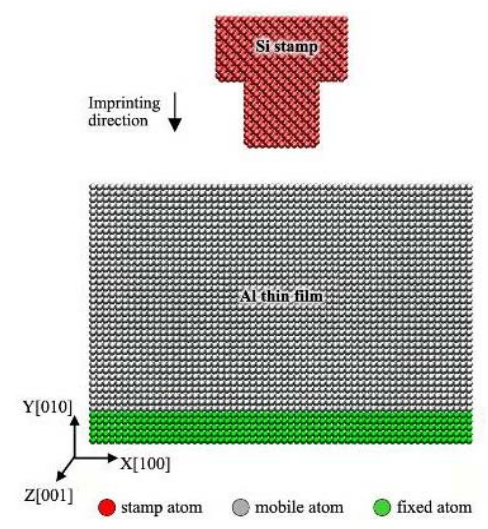

FIGURE I. MD MODEL OF NANOIMPRINT ON ALUMINIUM THIN FILM WITH SILICON STAMP

\section{RESULTS AND DISCUSSIONS}

\section{A. Deformation Mechanism during Nanoimprint}

Fig.2(a) shows the variation in force with moving distance of stamp during the loading stage in the nanoimprint process on aluminium thin film, which demonstrates there are four zones in the loading stage. Accordingly, Fig.3(a)presents representative MD snapshots of instantaneous defect structures obtained in the above four zones. In the first zone, the force is kept as zero until adhesion phenomena commenced between stamp and aluminium thin film, at which the force goes down to negative value. In the second stage, the force increases rapidly, but there is no dislocation observed, indicating the aluminium thin film undergoes elastic deformation. In the third zone, the force fluctuates largely due to dislocation nucleation and consequent glide of dislocations. In the final zone, the force reaches its maximum value when the stamp attains the maximum imprint depth.Fig.2(b) shows variation of force during the retraction stage.Fig.3(b) reveals that the dislocations glide towards surface and annihilate at top surface, which leads to material accumulation on the top surface.
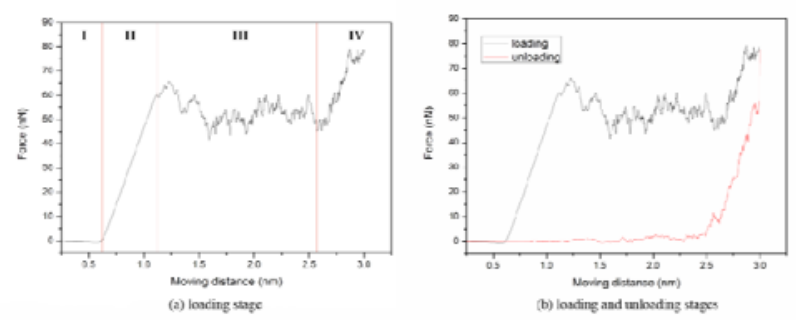

FIGURE II. CURVES OF FORCE VERSUS MOVING DISTANCE OF STAMP DURING NANOIMPRINT ON ALUMINIUM THIN FILM

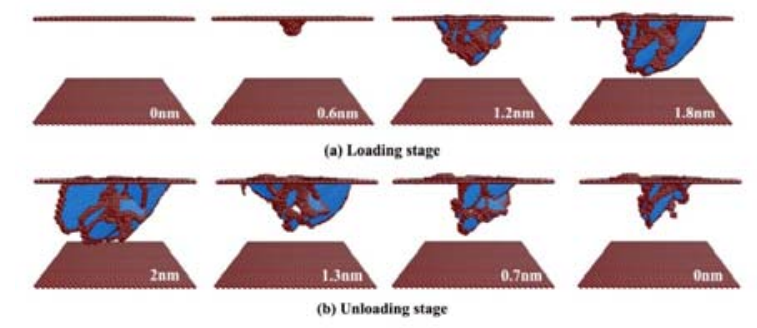

FIGURE III. MD SNAPSHOTS OF DEFECT STRUCTURES OBTAINED IN NANOIMPRINT PROCESSES ON ALUMINIUM THIN FILMS

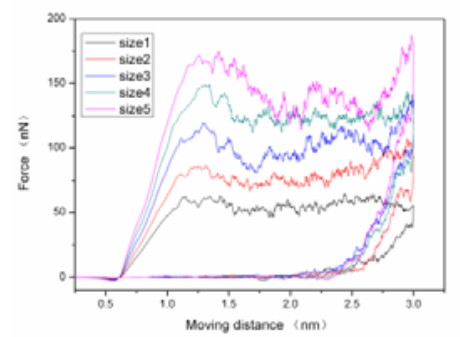

FIGURE IV. CURVES OF FORCE VERSUS MOVING DISTANCE OF STAMP DURING NANOIMPRINT ON ALUMINIUM THIN FILMS WITH DIFFERENT STAMP SIZES

\section{B. Effect of Stamp Size on the Nanoimprint Process}

MD simulations of nanoimprint of aluminium thin films with different stamp sizes are performed to investigate stamp size effect on nanoimprint process. On the basis of the original model, the length of silicon stamp is enlarged to 1.5 times, 2 times, 2.5 times and 3 times, while keeping the width unchanged.

Fig.4shows variations of force during nanoimprint on aluminium thin with different stamp sizes. It can be seen that characteristics of force variation are similar. Furthermore, both of the adsorption force and the force required for plastic deformation increase with the increasing of stamp size. The reason may be attributed that with the increasing of stamp size, the quantity of deformed atoms increases, which leads to the increase of the force required for plastic deformation. Fig.5 (a) shows MD snapshots of instantaneous defect structures at final zone of loading stage in nanoimprint on aluminium thin films, which shows that the range of dislocation increases significantly with the increase of stamp size. Fig.5 (b) shows MD snapshots of defect structures at final zone of retraction 
stage, and Fig.5 (c) shows surface morphology after imprinting. It is found that with the increase of stamp size, the range of defect structure increases significantly and surface deformation is also more serious.
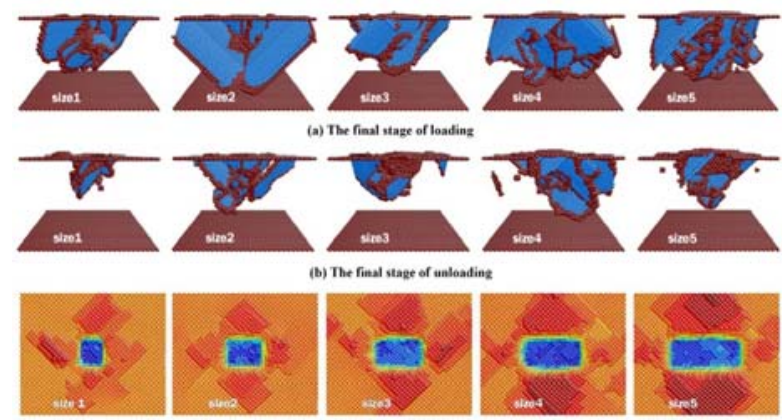

(b) The final stage of unloading
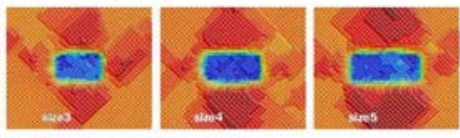

FIGURE V. MD SNAPSHOTS OF INSTANTANEOUS DEFECT STRUCTURES AND SURFACE MORPHOLOGY IN NANOIMPRINT ON ALUMINIUM THIN FILMS WITH DIFFERENT STAMP SIZES

\section{Effect of Stamp Shape on the Nanoimprint Process}

On the basis of the original model, different shapes of silicon stamp are considered to investigate stamp shape effect on nanoimprint process, as shown in Fig.6.Fig.7 shows curves of force during nanoimprint on aluminium thin films with different stamp shapes. While there is obvious adsorption phenomenon for the cylindrical stamp approaching the aluminium thin film, the adsorption phenomenon is less pronounced for the spherical stamp, and even disappears for the conical stamp. This discrepancy in adsorption phenomenon for different stamp shapes can be attributed to the different premier contact area of stamp with aluminium thin film, as the adsorption phenomenon increases with the increasing of the premier contact area. It also can be found from Fig.7 that the critical force required for plastic deformation initiation is also strongly dependent on stamp shape, and the order is: the cylindrical stamp is the largest, followed by spherical stamp, and the least is the conical stamp. This can be attributed to that the force required for plastic deformation initiation increases with the increasing of nanoimprinting volume between stamp and aluminium thin film. In other words, the quantity of deformed atoms increases when nanoimprint volume of stamps is increasing, which leads to the increase of the force required for plastic deformation. Fig.8 presents representative MD snapshots of instantaneous defect structures at different imprinting depth with different stamp shapes. It is found that the range of dislocation significantly increases with the increase of nanoimprinting volume of stamps, which is consistent with the change of the force as shown in Fig.7.Fig.9 further shows surface morphology after imprinting with different stamp shapes, which suggests that the range of defect structure increases significantly and surface deformation are also more serious with the increase of the volume of stamps.

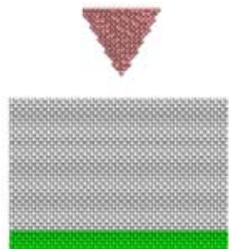

(a) cone

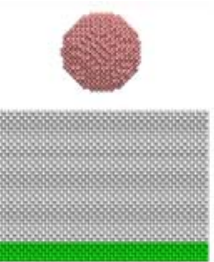

(b) sphere

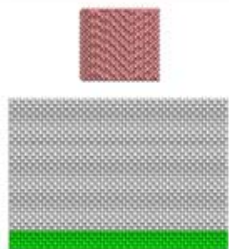

(c)cylinder
FIGURE VI. MD MODEL OF NANOIMPRINT ON ALUMINIUM THIN FILM WITH DIFFERENT SHAPES OF SILICON STAMP

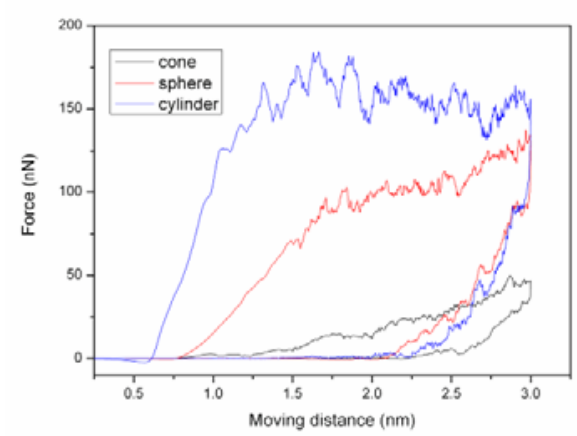

FIGURE VII. CURVES OF FORCE VERSUS MOVING DISTANCE OF STAMP DURING NANOIMPRINT ON ALUMINIUM THIN FILMS WITH DIFFERENT STAMP SHAPES

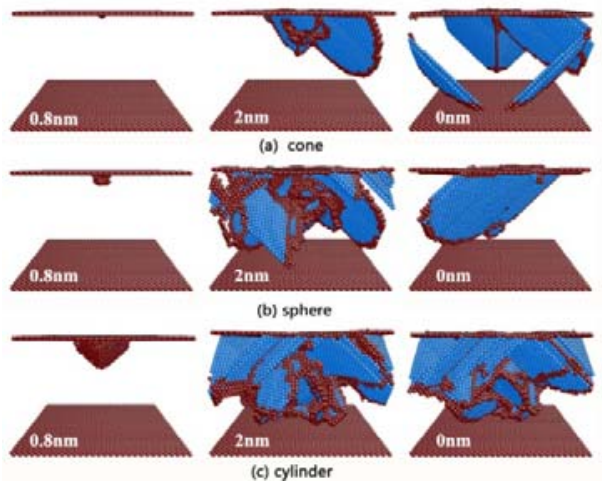

FIGURE VIII. MD SNAPSHOTS OF INSTANTANEOUS DEFECT STRUCTURES AT DIFFERENT IMPRINT DEPTHS DURING NANOIMPRINT ON ALUMINIUM THIN FILMS WITH DIFFERENT STAMP SHAPES
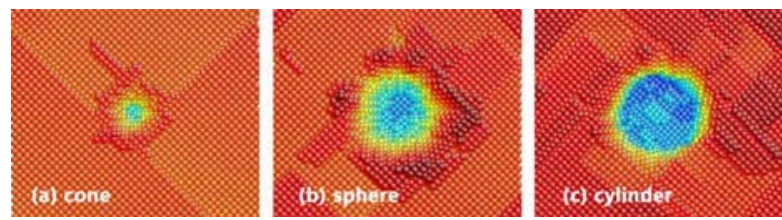

FIGURE IX. MD SNAPSHOTS OF SURFACE MORPHOLOGY AFTER NANOIMPRINT ON ALUMINIUM THIN FILMS WITH DIFFERENT STAMP SHAPES

\section{CONCLUSIONS}

MD simulations of nanoimprint of single crystal aluminium thin films are performed, and effects of stamp size and stamp shape on plastic deformation are especially 
emphasized. Simulation results suggest that plastic deformation of the aluminium thin film is governed by dislocation activity. Stamp size and stamp shape have significant influence on the dislocation movements and imprint forces during the nanoimprint processes. The critical force required for plastic deformation initiation increases with the increasing of nanoimprinting volume of stamps with aluminium thin film, which is strongly dependent on stamp size and shape. It is found that the adsorption phenomenon of force variation is closely related to the premier contact area between stamp and aluminium thin film, the increase of which is accompanied with the increase of the adsorption phenomenon.

\section{REFERENCES}

[1] S.Y. Chou, P.R. Krauss, P.J. Renstrom, Imprint of sub-25 nm vias and trenches in polymers. Applied Physics Letters.67 (1995) 3114.

[2] Q.C. Hsu, C.D. Wu, T.H. Fang, Studies on nanoimprint process parameters of copper by molecular dynamics analysis. Comput. Materials Science. 34 (2005) 314-322.

[3] C.H. Yao, C.H. Chang, C.W. Hsieh, C.K. Sung, Effects of mold shape and sidewall roughness on nanoimprint by molecular dynamics simulation. Microelectronic Engineering. 87 (2010)864-868.

[4] D.W. Cheng, H.F. Te, C.C. Chi, M.K. Li, Effect of mold geometry on nanoformed aluminium films investigated using molecular dynamics simulations. Computational Material Science. 74(2013)17-22.

[5] D.W. Cheng, C.C. Po-Yuan, C.C. Chia, et al. Effect of water separation layer on metal nanoforming process investigated using molecular dynamics simulations. Journal of Applied Physics.114, 174303(2013).

[6] S.M. Foiles, M.I. Baskes, M.S. Daw, Embedded-atom-method functions for the FCC metals $\mathrm{Cu}, \mathrm{Ag}, \mathrm{Au}, \mathrm{Ni}, \mathrm{Pd}$,Pt and Other Alloys. Physical Review B.33(1986) 7983-7991.

[7] Y. Yuan, T. Sun, J. Zhang, Y. Yan, Molecular dynamics study of void effect on nanoimprint of single crystal aluminum. Applied Surface Science.257 (2011) 7140-7144.

[8] Y.S. He, T. Sun, Y. Yuan, et al. Molecular dynamics study of the nanoimprint process on bi-crystal Al thin films with twin boundaries. Microelectronic Engineering. 95 (2012) 116-120. 